Pathologe 2019 · 40:13-20

https://doi.org/10.1007/s00292-019-0568-5

Online publiziert: 1. Februar 2019

(c) Der/die Autor(en) 2019

\section{Redaktion}

H. A. Baba, Essen

CrossMark

\section{S. F. Lax}

Institut für Klinische Pathologie und Molekularpathologie, LKH Graz II, Standort West, Akademisches Lehrkrankenhaus der Medizinischen Universität Graz, Graz, Österreich

\title{
Vorläuferläsionen der Endometriumkarzinome
}

Der Großteil der Endometriumkarzinome, im speziellen die endometrioiden Karzinome und seine Varianten, entwickelt sich über eine definierte Vorstufe, die atypische Endometriumhyperplasie, die wiederum auf dem Boden einer Endometriumhyperplasie ohne Atypien entsteht [1]. Die WHO hat 2014 die Klassifikation der Präneoplasien des endometrioiden Endometriumkarzinoms durch die Reduktion auf 2 Kategorien wesentlich vereinfacht [2]. Eine Gegenüberstellung mit der WHO-Klassifikation 2003 und der traditionellen deutschsprachigen Einteilung nach Frau Prof. Dallenbach-Hellweg [3] findet sich in - Tab. 1, wobei es eine Überschneidung zwischen den einzelnen Untergruppen gibt.

\section{Ursachen für eine Endo- metriumhyperplasie}

Eine wesentliche Ursache für eine Endometriumhyperplasie ist der Hyperöstrogenismus, charakterisiert durch einen Überschuss an Östrogenen ohne entsprechendes Ausmaß des Gegenspielers Progesteron [4]. Dieser Überschuss wird entweder endogen produziert oder exo-

\section{Infobox 1 Formen der Metaplasie}

\footnotetext{
- Muzinöse Metaplasie

- Plattenepitheliale Metaplasie

- Sekretorische Metaplasie

- Eosinophile Metaplasie

- Ziliäre Metaplasie

- Papilläre synzytiale Metaplasie

- Papilläre Proliferationen
}

gen durch Medikation zugeführt. Ein endogener Überschuss an Östrogenen ist typisch für die Perimenopause, in der es zur Reifung von Follikeln ohne Eisprung kommt. Auch durch eine übermäßige Umwandlung von Dehydroepiandrosteron (DHE) durch Aromatase im überschüssigen Fettgewebe übergewichtiger Frauen kann sich ein Hyperöstrogenismus ausbilden. Weitere Ursachen einer übermäßigen endogenen Östrogenproduktion sind z. B. Granulosazelltumoren des Ovars [5]. Die Endometriumhyperplasie ohne Atypien (EH) geht aus einer protrahierten, ungeordneten Proliferation (engl. „disordered proliferative phase") hervor (• Abb. 1) und ist durch eine Vermehrung von Drüsen und Stroma charakterisiert, wobei die Drüsen unterschiedlich dicht stehen können (• Abb. 2). Die Gewebearchitektur kann einerseits einem sog. Schweizer-KäseMuster entsprechen, andererseits durch dicht liegende Drüsen eine erhöhte Komplexität aufweisen [2]. Die EH stellt per se keine Präkanzerose dar, ist aber mit einem etwa 3- bis 4fachen Risiko für die Entstehung eines Endometriumkarzinoms verbunden. Laut Literatur gehen etwa 1-3\% aller Endometriumhyperplasien ohne Atypien in ein Karzinom über [6].

\section{Atypische Endometrium- hyperplasie}

Für die atypische Endometriumhyperplasie (AEH) wird seit der WHO 2014 auch das Synonym einer endometrioiden intraepithelialen Neoplasie (EIN) verwendet [2]. Es wurden dabei Kriterien des EIN-Systems mit der ursprünglichen WHO-Klassifikation fusioniert und die Bezeichnung endometrial (auf deutsch übersetzt: „das Endometrium betreffend") durch endometrioid ersetzt. Die AEH/EIN ist in der Regel durch eine komplexe Drüsenarchitektur charakterisiert, wobei zwischen den Drüsen nur wenig Stroma vorhanden ist (• Abb. 3). Das Drüsenepithel besteht aus zytoplasmareichen, großen Zellen mit ungeordnet liegenden, meist blasigen Zellkernen mit Nukleolen. Mitunter sind die Zellkerne auch spindelförmig („Zigarrenform"), aber gegenüber der Proliferationsphase ungeordnet (• Abb. 4). Diese unregelmäßige Anordnung des

Tab. 1 Vergleich der aktuellen WHO-Klassifikation (2014) der Vorstufen des Endometriumkarzinoms mit früheren Klassifikationen

\begin{tabular}{|c|c|c|}
\hline $\begin{array}{l}\text { Klassifikation nach } \\
\text { Dallenbach-Hellweg }\end{array}$ & $\begin{array}{l}\text { WHO-Klassifikation } \\
\text { 1994/2003 }\end{array}$ & $\begin{array}{l}\text { WHO-Klassifikation } \\
2014\end{array}$ \\
\hline $\begin{array}{l}\text { Glandulär-zystische } \\
\text { Hyperplasie } \\
\text { Adenomatöse Hy- } \\
\text { perplasie } \\
\text { Grad } 1\end{array}$ & $\begin{array}{l}\text { Einfache Hyperplasie ohne Atypien } \\
\text { Komplexe Hyperplasie ohne Atypien }\end{array}$ & $\begin{array}{l}\text { Endometriumhyperplasie ohne } \\
\text { Atypien }\end{array}$ \\
\hline \multicolumn{3}{|l|}{ Grad 2} \\
\hline Grad 3 & $\begin{array}{l}\text { Atypische Endometriumhyperplasie } \\
\text { (einfach bzw. komplex) }\end{array}$ & $\begin{array}{l}\text { Atypische Endometriumhyperpla- } \\
\text { sie/endometrioide intraepitheliale } \\
\text { Neoplasie (AEH/EIN) }\end{array}$ \\
\hline
\end{tabular}




\section{Schwerpunkt: Gynäkopathologie}
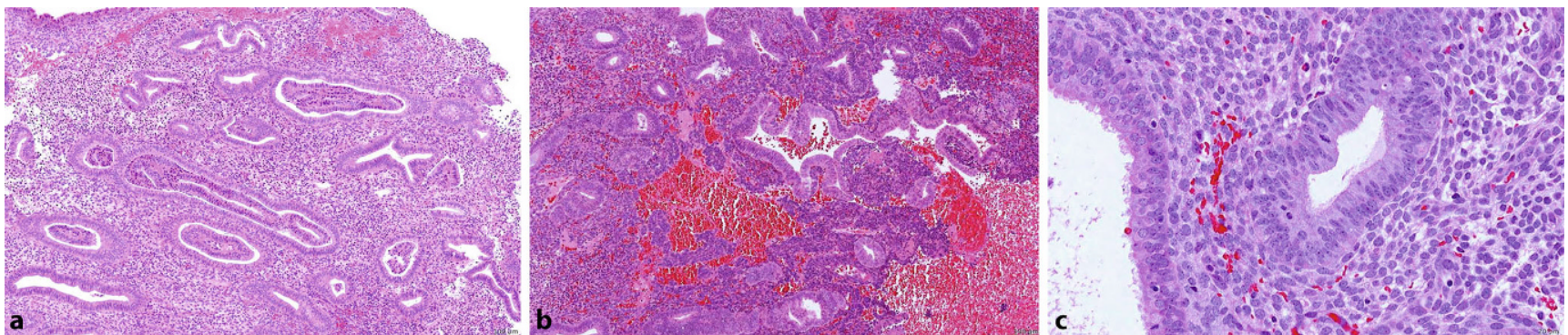

Abb. 1 A Unregelmäßig proliferiertes Endometrium mit ausgeprägtem Teleskopphänomen (a) und Zeichen vorzeitiger Abstoßung (b). Das Drüsenepithel ist pseudostratifiziert und enthält Mitosen als Ausdruck der Proliferation (c). HE-Färbung
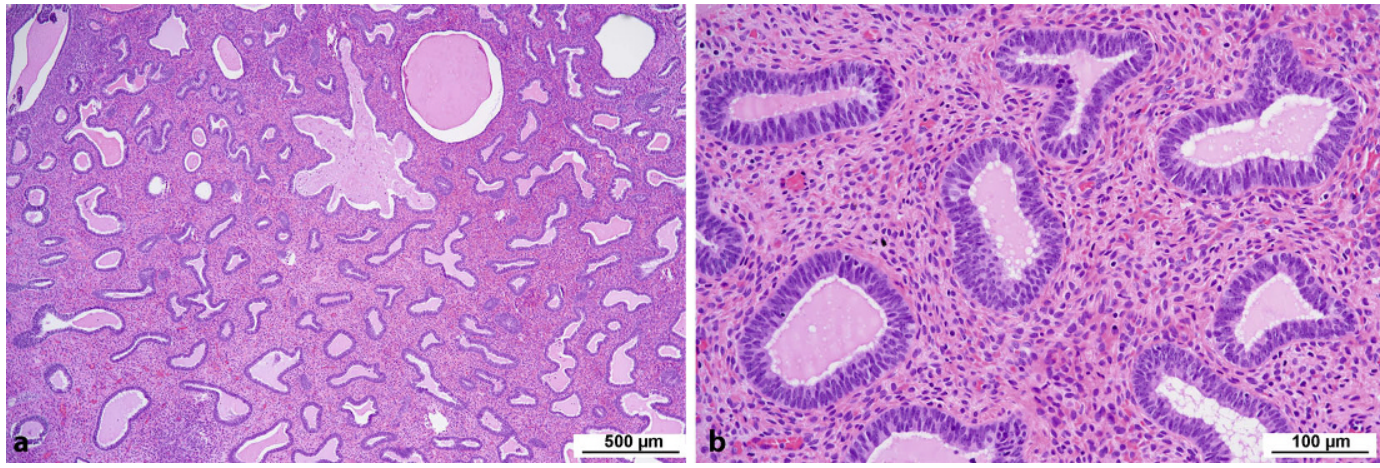

Abb. $2<$ Endometriumhyperplasie ohne Atypien. Drüsen und Stroma sind vermehrt, die Drüsen zystisch und verzweigt (a), das Drüsenepithel unauffällig (b). HE-Färbung
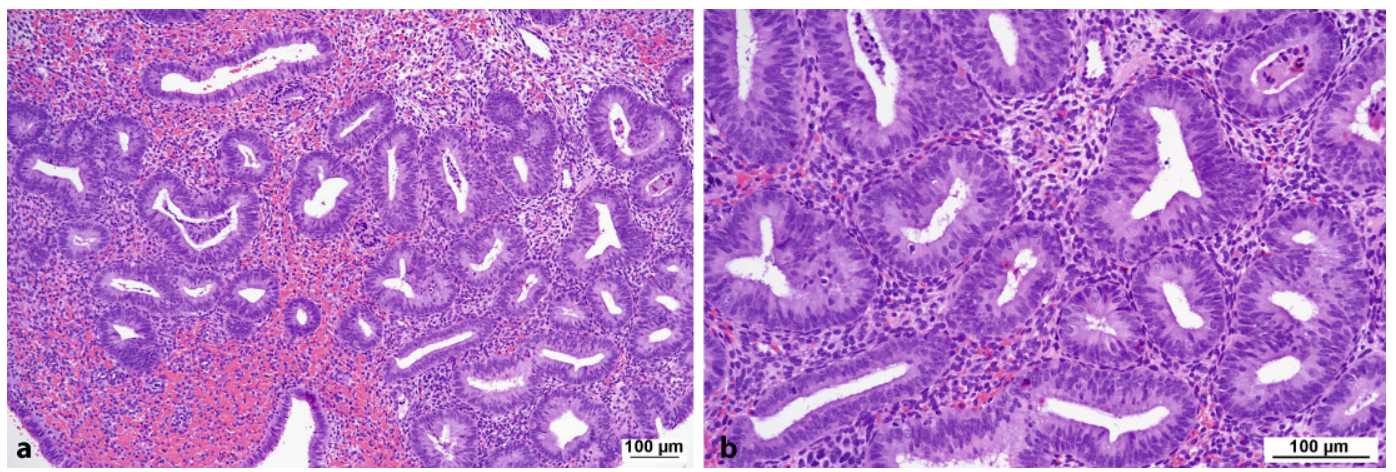

Abb. $3 \Delta$ Atypische Endometriumhyperplasie/endometrioide intraepitheliale Neoplasie (AEH/EIN). Der Stromagehalt ist innerhalb einer bestimmten Fläche gegenüber den Drüsen deutlich vermindert (a), das Drüsenepithel atypisch mit ungeordnet liegenden runden bis ovalen Zellkernen. Im Vergleich dazu eine normale Drüse im linken unteren Bildbereich (b). HE-Färbung
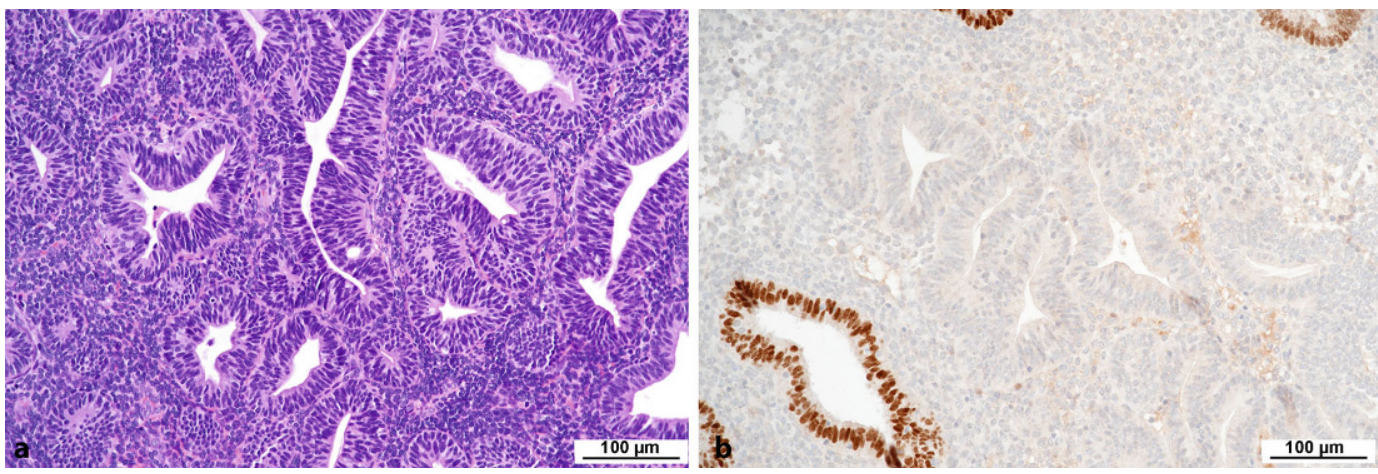

Abb. $4<$ Atypische Endometriumhyperplasie/ endometrioide intraepitheliale Neoplasie (AEH/EIN) mit „zigarrenförmigen"Zellkernen (a HE-Färbung) und Verlust der Immunreaktivität für PAX2, welche in den normalen Drüsen erhalten ist (b, DAB-Färbung) 
Drüsenepithels wird auch als Verlust der Polarität bezeichnet. Ein wesentliches neues Kriterium, das dem ursprünglichen EIN-System entnommen wurde, ist der Unterschied zwischen den atypischen Drüsen und quasi „normalen“ entweder inaktiven/atrophen oder zyklisch veränderten Drüsen in der Umgebung (www. endometrium.org). Problematisch ist die diagnostische Subjektivität bei der Beurteilung und Einstufung der Drüsenveränderungen, im Speziellen der Atypien. Die AEH/EIN ist als echte Neoplasie anzusehen, die zum Großteil klonal ist und genetische Veränderungen aufweist [7]. Dazu zählen eine Inaktivierung von $P T E N$ oder $P A X 2$, eine Aktivierung von KRAS und Beta-Catenin durch Mutation sowie eine Mikrosatelliteninstabilität durch Methylierung des MismatchRepair(MMR)-Proteins MLH1 [8, 9]. Neben einem Hyperöstrogenismus gilt für die Entstehung der AEH/EIN als wesentliche Ursache eine hereditäre Komponente, insbesondere Keimbahnmutationen in entscheidenden Genen, wie z.B. in den MMR-Proteinen im Rahmen des Lynch-Syndroms oder von PTEN beim Cowden-Syndrom. Das Risiko einer $\mathrm{AEH} / \mathrm{EIN}$, in ein Karzinom überzugehen, ist gegenüber Patientinnen mit normalem Endometrium um das bis zu 45-fache erhöht [10]. Zudem weist bis zu einem Drittel aller Frauen mit der Diagnose einer AEH/EIN in der Biopsie oder Kürettage bereits ein Endometriumkarzinom auf, wenn der Uterus innerhalb weniger Monate operativ entfernt wird [11]. Dennoch gehen nicht alle AEH/EIN in ein Karzinom über und es fehlen verlässliche prädiktive Parameter, insbesondere Biomarker. Die Ergebnisse für PTEN waren in dieser Hinsicht negativ [12]. Ein mögliches prädiktives Kriterium für ein assoziiertes Karzinom ist das Vorhandensein kribröser Strukturen [13].

\section{Metaplasie: Terminalezelluläre Differenzierung}

Im Rahmen der Endometriumhyperplasie und der AEH/EIN treten häufig metaplastische Veränderungen auf (• Abb.5), die diagnostisch anspruchsvoll sein können [14]. Die verschiedenen Metaplasie-

Pathologe 2019 · 40:13-20 https://doi.org/10.1007/s00292-019-0568-5

(c) Der/die Autor(en) 2019

\section{S. F. Lax}

\section{Vorläuferläsionen der Endometriumkarzinome}

\section{Zusammenfassung}

Die WHO-Klassifikation 2014 unterscheidet 2 Vorläuferläsionen für das endometrioide Endometriumkarzinom: die Endometriumhyperplasie ohne Atypien (EH) und die atypische Endometriumhyperplasie/endometrioide intraepitheliale Neoplasie (AEH/EIN). Die $\mathrm{AEH} / \mathrm{EIN}$ ist durch dicht liegende Drüsen mit atypischem Epithel charakterisiert, zwischen denen das Stroma deutlich reduziert ist. Die zellulären Atypien sind durch Verlust der Polarität mit Ausbildung runder bis ovaler Zellkerne mit deutlichen Nukleolen charakterisiert. Der Vergleich mit quasi normalen Drüsen der Umgebung erleichtert das Erkennen der Atypien. Die AEH/EIN kann sekretorische und muzinöse Veränderungen sowie eine Plattenepithelmetaplasie enthalten. Epitheliale Metaplasien können sowohl in benignen Läsionen als auch in Karzinomen des Endometriums vorkommen. In etwa zwei Drittel der AEH/EIN kommt es zu einem Verlust der Immunreaktivität für PTEN und/oder PAX2. Die Differenzialdiagnose zum invasiven, gut differenzierten endometrioiden Karzinom basiert auf einem konfluenten Wachstumsmuster mit Ausbildung labyrinthartiger oder kribröser Strukturen. Differenzialdiagnostisch spielen hyperplastische Polypen und papilläre Proliferationen eine Rolle, andererseits kann eine AEH/EIN auch in Polypen vorkommen. Eine Therapie einer AEH/EIN mit Gestagenen ist nicht evidenzbasiert, führt aber häufig zu deren Rückbildung. Das seröse intraepitheliale Karzinom (SEIC) ist durch hochgradige zelluläre Atypien und Polymorphie, Ablösen von Zellen, ein mutationspezifisches immunhistchemisches Färbemuster für P53 und einen erhöhten Ki67 Färbeindex charakterisiert. Obwohl es Vorläufer des serösen Karzinoms ist, wird das SEIC biologisch als nichtinvasives seröses Karzinom betrachtet, da es bereits mit extrauterinen Metastasen einhergehen kann.

Schlüsselwörter

Endometriumhyperplasie - Endometriumkarzinom · Humanes PTEN-Protein · Metaplasie . Seröses intraepitheliales Karzinom

\section{Precursor lesions of endometrial carcinoma}

\section{Abstract}

The 2014 WHO classification distinguishes between endometrial hyperplasia without atypia (EH) and atypical endometrial hyperplasia/endometrioid intraepithelial neoplasia (AEH/EIN). AEH/EIN is characterized by crowded glands with cytologically atypical epithelium separated by little intervening stroma. Cellular atypia is characterized by nuclear enlargement and rounding, pleomorphism, loss of polarity, and presence of nucleoli. The diagnosis of atypia is facilitated by comparison with areas of adjacent normal and non-atypical glands, respectively. $\mathrm{AEH} / \mathrm{EIN}$ is often associated with squamous but also secretory and mucinous metaplasia. Loss of PTEN and/or PAX2 immunoreactivity occurs in up to two thirds of AEH/EIN. In contrast, invasive low-grade endometrioid carcinoma shows confluent growth with loss of stroma and formation of labyrinth-like or cribriform structures. Differential diagnosis includes different forms of metaplasias, papillary proliferations, and hyperplastic polyps. Epithelial metaplasia may be present in various benign endometrial lesions as well as in endometrioid adenocarcinoma. AEH/EIN may also occur in endometrial polyps. Progestin therapy of AEH/EIN has low level of evidence but frequently leads to complete regression. Serous intraepithelial carcinoma (SEIC) is characterized by high-grade cellular atypia and polymorphism, detachment of cells, a mutant immunoreactive pattern for the P53 and an increased Ki67 labeling index. Although designated as precursor of serous carcinoma of the endometrium, biologically it is considered a non-invasive serous carcinoma since it may already be associated with massive extrauterine spread.

\section{Keywords}

Endometrial hyperplasia $\cdot$ Endometrioid carcinoma · Human PTEN protein · Metaplasia · Serous endometrial intraepithelial carcinoma 

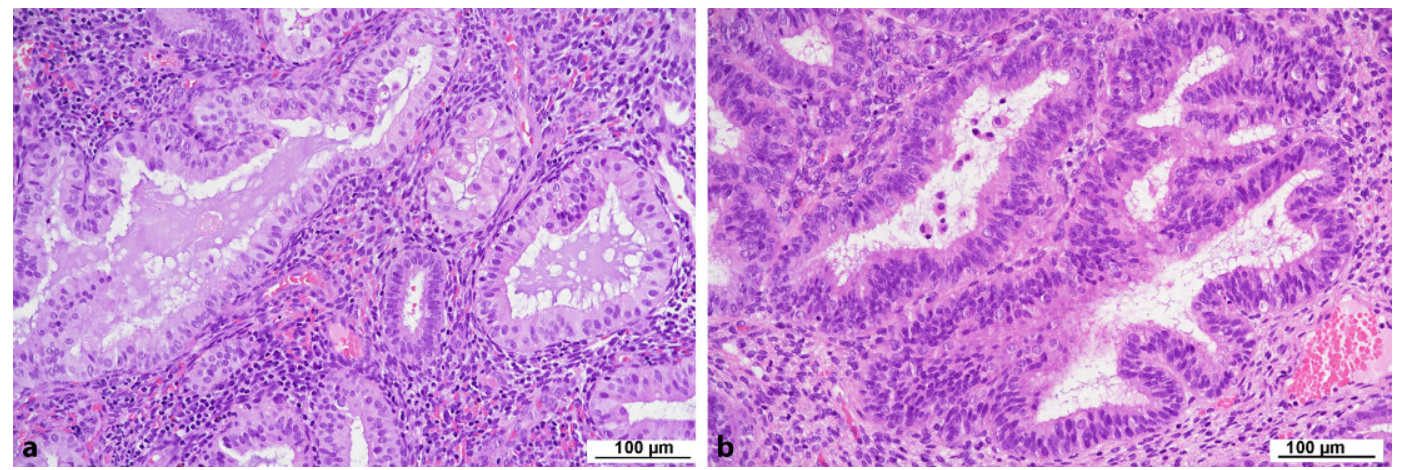

Abb. $5 \triangleleft$ Metaplastische Veränderungen in der atypischen Endometriumhyperplasie/endometrioide intraepitheliale Neoplasie (AEH/EIN), sekretorisch (a) bzw. tubenepithelartig (a,b). HE-Färbung
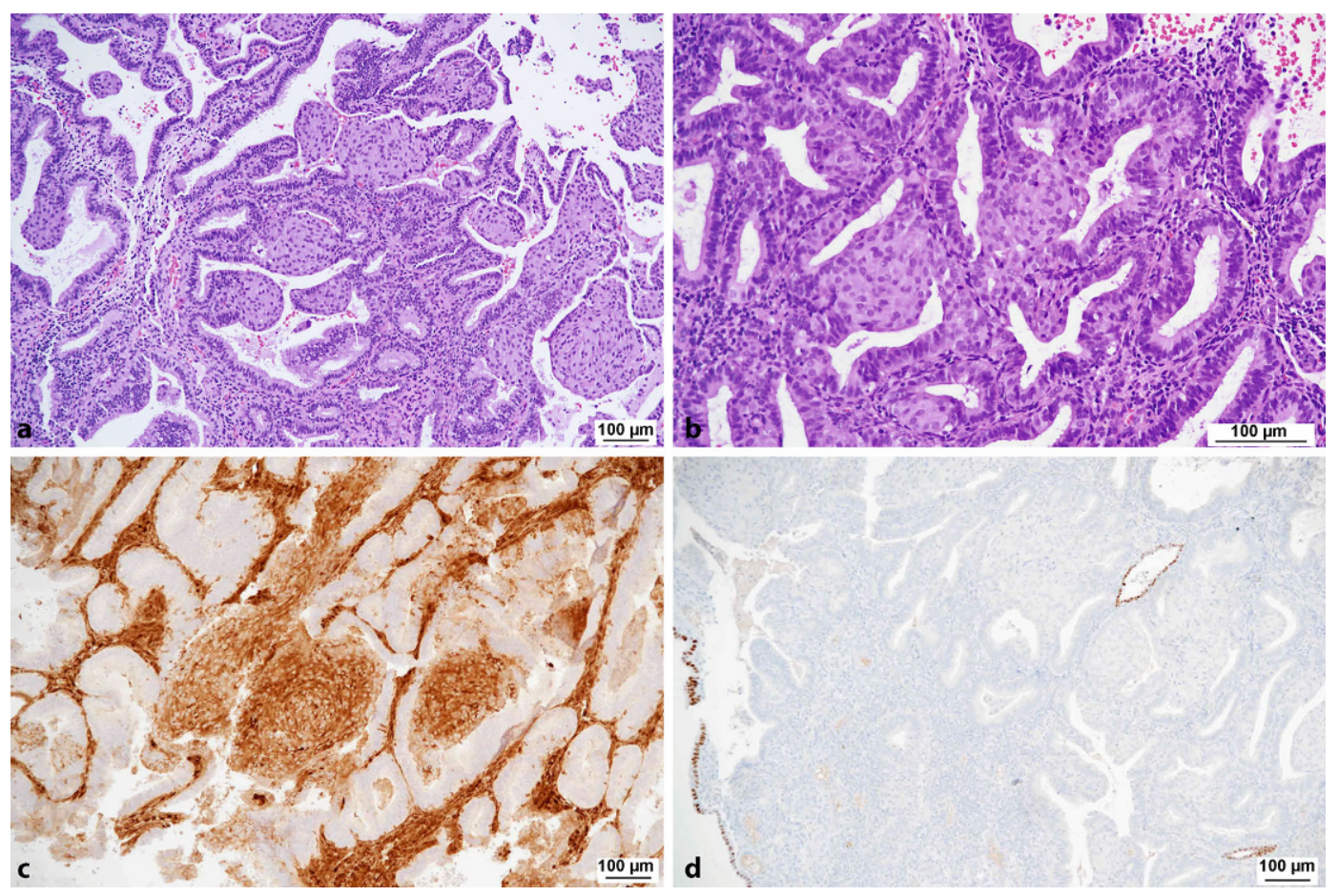

Abb. $6<$ Plattenepithelmetaplasie mit zytologisch blanden Morulae $(\mathbf{a}, \mathbf{b})$. Gleichzeitiger Verlust der Immunreaktivität von PTEN (c) und PAX2 (d). Die PTEN-Immunreaktivität ist in den Morulae erhalten. HE-Färbung $(a, b)$, DAB-Färbung $(c, d)$

typen sind in $\bullet$ Infobox 1 aufgelistet. Metaplasien werden als Ausdruck terminaler Differenzierung betrachtet und haben für sich keine klinische Signifikanz; diese wird durch die zugrunde liegende Veränderung beeinflusst [15]. Oft findet sich dabei eine Mischung mehrerer Metaplasietypen. Einige, wie z. B. die plattenepitheliale Metaplasie (• Abb. 6), sind häufig mit einer AEH/EIN vergesellschaftet und funktionell inaktiv [16]. Wenn sich muzinöse und sekretorische Veränderungen in einer AEH/EIN finden, sollte dies nicht als sekretorische Transformation des Endometriums fehlinterpretiert werden. Atypische muzinöse Läsionen sind in beinahe der Hälfte der Fälle mit einem invasiven Adenokarzinom vergesellschaftet, wenn innerhalb von weniger als 3 Monaten eine Hysterektomie durchgeführt wird [17]. Die diagnostische Reproduzierbarkeit muzinöser Läsionen in Abradaten und Endometriumbiopsien ist selbst unter spezialisierten Gynäkopathologen suboptimal [18].

Im Gegensatz zu AEH/EIN ist das seröse intraepitheliale Karzinom (SEIC) nicht mit Metaplasien vergesellschaftet, muss aber gegenüber einzelnen Metaplasietypen abgrenzt werden. Die papilläre synzytiale Metaplasie (• Abb. 7 und 8) ist zum Teil Ausdruck einer Regeneration ohne erhöhtes Karzinomrisiko und zytologisch meist bland, kann aber immunhistochemisch eine Überexpression von TP53 aufweisen. Sie darf daher nicht mit einem SEIC verwechselt werden [19]. Auch eine Tubenepithelmetaplasie mit zytologischen Atypien kann histologisch einem SEIC ähneln, unterscheidet sich von diesem aber immunhistochemisch durch einen niedrigen Ki-67-Färbeindex und eine Wildtyp-Immunreaktivität für TP53 ähnlich der Tubenepithelmetaplasie ohne Atypien [20]. Seltener kommen im hyperplastischen Endometrium papilläre Proliferationen mit meist muzinösem Epithel vor, die ein komplexes Muster und zelluläre Atypien aufweisen können (• Abb. 8). Dabei wird auch von komplexen papillären Läsionen bzw. komplexen atypischen papillären Läsionen gesprochen, die zum Teil einer weiteren histologischen Abklärung bedürfen [21]. Insbesondere papilläre muzinöse Läsionen mit Atypien können mit meist gut differenzierten Karzinomen assoziiert sein [22]. 

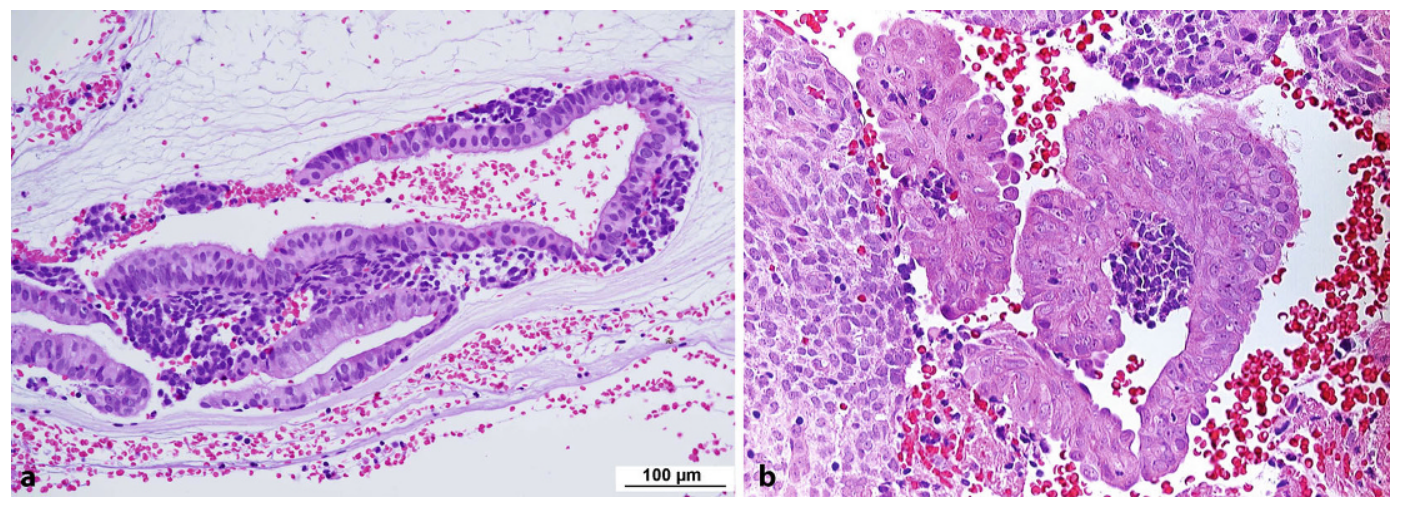

Abb. $7<$ Differenzialdiagnostisch zu beachten sind die zytologisch blande Tubenepithelmetaplasie, hier in einem unregelmäßig sekretorisch transformierten Endometrium (a) und die papilläre synzytiale Metaplasie (b), jeweils bei vorzeitiger Abstoßung. HE-Färbung
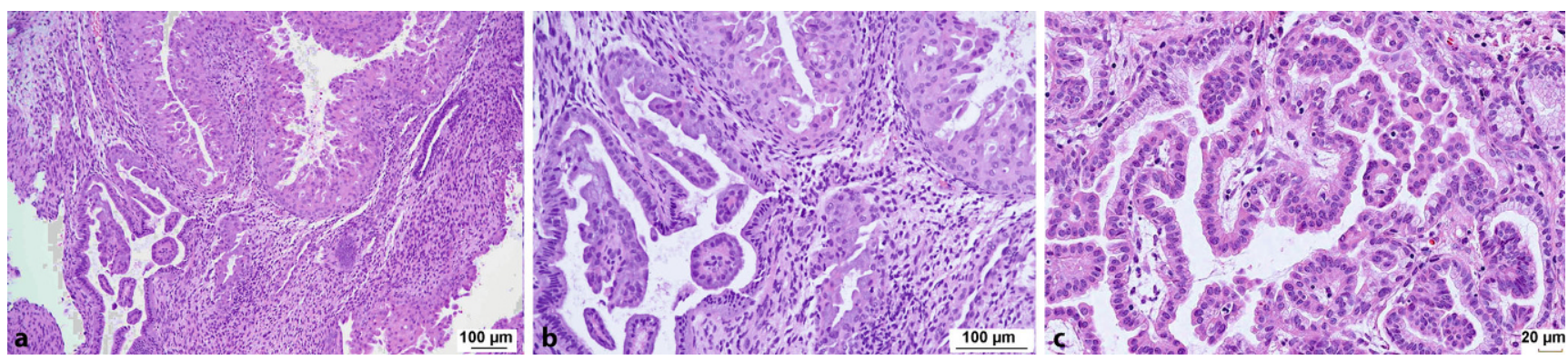

Abb. 8 ム Papilläre muzinöse (links unten) bzw. synzytiale (rechts oben) Metaplasie mit komplexer Architektur (a), jedoch blander Zytologie (b). Atypische papilläre muzinöse Proliferation mit stärkerer Komplexität der Papillen und geringen bis mäßigen Atypien (c). HE-Färbung

\section{Diagnostische Biomarker}

Im Zuge der Progression der Endometriumhyperplasie zur AEH/EIN und weiter zum endometrioiden Karzinom kommt es zu Mutationen und anderen Veränderungen in wesentlichen Genen, wie z.B. PTEN, die meist mit einer Inaktivierung und dem konsekutiven Verlust der Proteinexpression einhergehen. Dennoch gibt es bis dato weder für die Differenzialdiagnose zwischen einer Endometriumhyperplasie ohne Atypien und einer AEH/EIN noch zwischen der AEH/EIN und dem endometrioiden Karzinom G1 spezifische Biomarker. Wissenschaftlich untersucht wurden zwar insbesondere PTEN und PAX2 (- Abb. 4 und 6), deren Inaktivierung und Verlust der Immunreaktivität bei der Diagnostik von AEH/EIN hilfreich sein können, aber nicht beweisend sind [8]. Die Evidenz für diese Marker ist begrenzt. In etwa $70 \%$ der AEH/EIN kommt es im Gegensatz zur Hyperplasie ohne Atypien zu einer klonalen Expansion von PTEN- bzw. PAX2-negativen Drüsen. Dies hilft bei der Abschätzung diagnostisch schwieriger Fälle, die Dia- gnose sollte dennoch immer auf Basis der Histomorphologie erfolgen. Etwa ein Drittel der AEH/EIN weist eine intakte Immunreaktivität für PTEN auf. In endometrioiden Karzinomen liegt der Verlust der Immunreaktivität für PTEN bzw. PAX2 bei etwa $85 \%$. Schließlich könnte auch das Mismatch-Repair-Protein MLH1 immunhistochemisch für die Differenzialdiagnose zwischen Endometriumhyperplasie ohne Atypie und AEH/EIN herangezogen werden. Etwa ein Viertel der Endometriumkarzinome weist meist infolge eines MLH1-Verlustes durch Promotormethylierung eine Mikrosatelliteninstabilität auf und diese Veränderungen können bereits in der benachbarten AEH/EIN nachgewiesen werden [23].

\section{Differenzialdiagnose zum endometrioiden Karzinom}

Die Differenzialdiagnose zwischen einer AEH/EIN und einem gut (hoch) differenzierten endometrioiden Karzinom (EC G1) basiert auf einer Reihe von Kriterien, unter denen der Verlust des Stromas eines der wesentlichen darstellt [24].
Dieser resultiert in einem konfluenten glandulären oder kribrösen Muster, das auch als labyrinthartig bezeichnet wird (- Abb.9). Eine desmoplastische Stromareaktion ist Ausdruck eines infiltrativen Wachstums, findet sich aber nur in einem Teil der Fälle. Andererseits kann die Abgrenzung zum fibrotischen Stroma von Polypen schwierig sein. Eine komplexe papilläre Architektur ist zwar typisch für Karzinome, die alleinige Präsenz von $\mathrm{Pa}$ pillen ist aber für eine Karzinomdiagnose nicht ausreichend [13].

\section{Veränderungen einer AEH/EIN nach Gestagentherapie}

Für Fälle mit AEH/EIN oder EC G1 bietet sich bei Wunsch nach Erhaltung des Uterus die therapeutische Option einer Gestagentherapie, beispielsweise mit einer gestagenhaltigen Spirale. Für diesen therapeutischen Ansatz liegt jedoch nur ein geringer Evidenzlevelvor, da prospektiv randomisierte Studien fehlen. Daher wurden in die S3-Leitlinien der Deutschen Krebsgesellschaft nur konsensbasierte Empfehlungen aufgenommen [25]. Die publizierten Metaanalysen kommen 

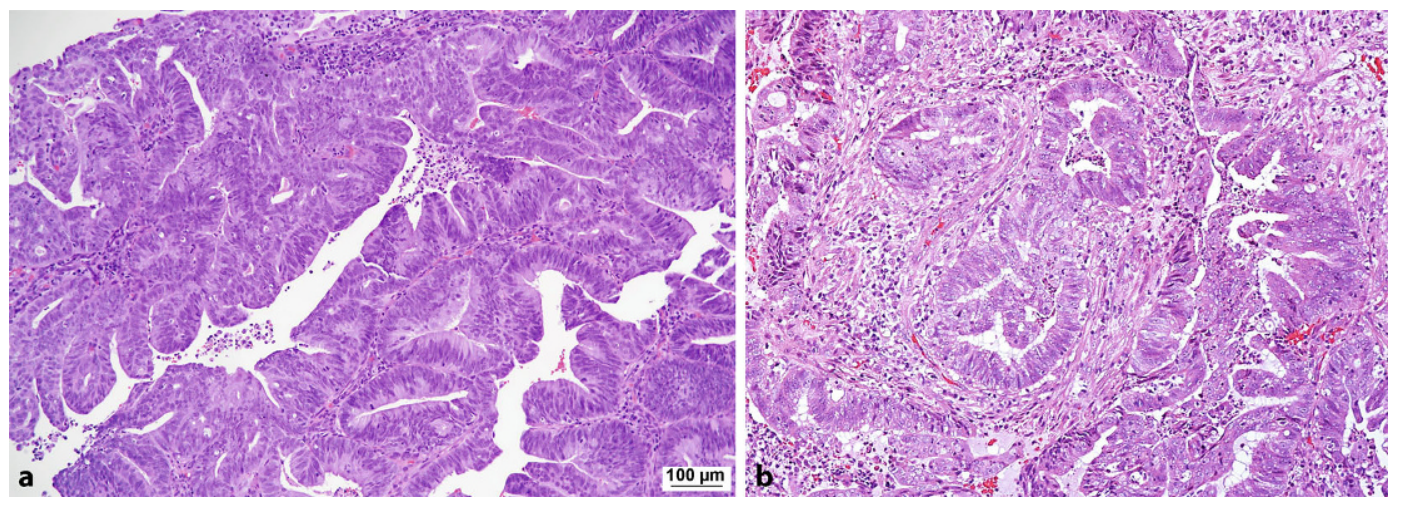

Abb. $9 \triangleleft$ Gut differenziertes endometrioides Karzinom mit labyrinthartiger Architektur (a) bzw. unregelmäßigen Drüsen in desmoplastischem Stroma (b).
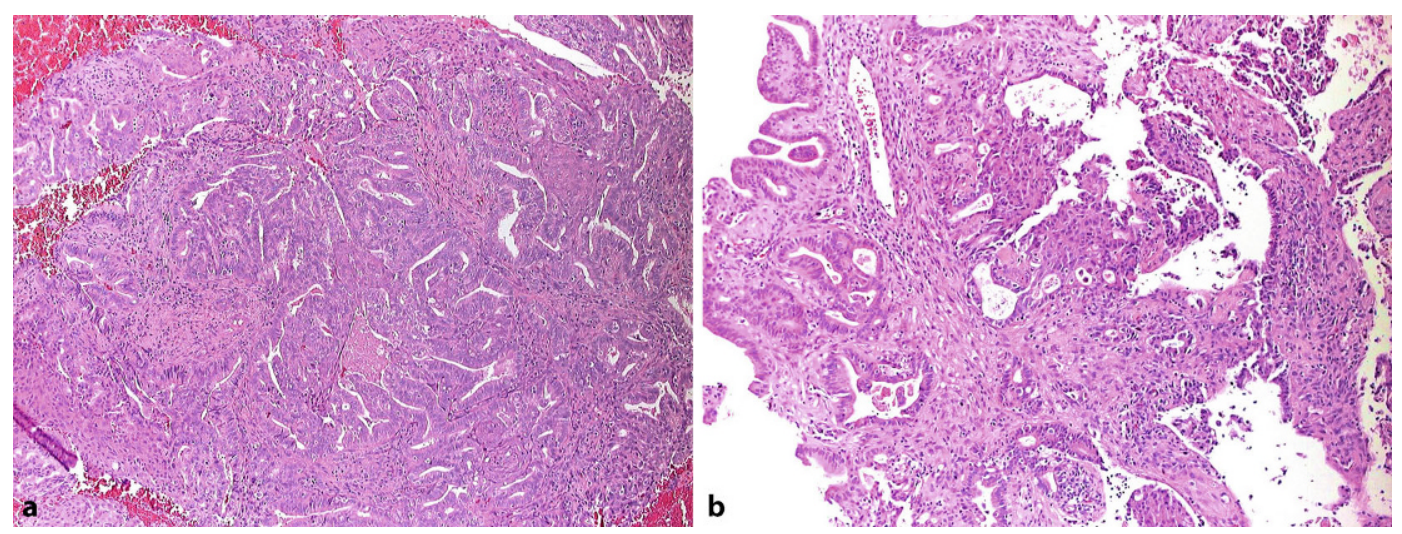
HE-Färbung

Abb. 10 ॥ Gut differenziertes endometrioides Karzinom und atypische Endometriumhyperplasie/endometrioide intraepitheliale Neoplasie AEH/EIN (a) mit partieller Remission nach 5-monatiger Therapie mit einem oralen Gestagen (500 mg Medroxyprogesteron/Tag). Man beachte die geringere Zellularität der Drüsen und die deziduaartige Stromareaktion im Zuge der Therapie, ein konfluentes Drüsenwachstum ist jedoch erhalten (b). Ein vorzeitiger Therapieabbruch führtezu einer Tumorprogression. HE-Färbung

unter Einschluss derselben Publikationen zum Teil zu unterschiedlichen Ergebnissen. Es wird empfohlen, die Zweitmeinung eines mit der Thematik besonders vertrauten Pathologen einzuholen [25]. Die meisten Arbeiten, darunter jene mit solider histopathologischer Analyse der Fälle, schließen sowohl AEH/EIN als auch EC G1 mit ein. Eine Rückbildung fand sich in retrospektiven Studien in bis zu mehr als $70 \%$ der AEH/EIN, wobei sich ein Therapieerfolg in Form einer Rückbildung der AEH/EIN spätestens nach 9 Monaten einstellen sollte. Der Therapieverlauf sollte mittels Pipelle oder Kürettage kontrolliert werden. Im Zuge der Gestagentherapie kommt es zu einer verminderten Ratio zwischen Drüsen und Stroma ( $\bullet \mathbf{A b b}$. 10), einer verminderten mitotischen Aktivität, einem Verlust der zellulären Atypie und zu einer Reihe metaplastischer Veränderungen des Drüsenepithels (muzinöse, sekretorische, eosinophile und plattenepi- theliale Veränderungen) [26]. Die Architektur der Läsionen verändert sich erst nach länger andauernder Gestagentherapie. Andererseits können auch kribriforme und papilläre Veränderungen durch Gestagene induziert werden, die nicht als Tumorprogression interpretiert werden sollten. Eine verminderte Zellularität der Drüsen ist der stärkste Prädiktor einer kompletten Remission nach Abschluss der Therapie. Dem Status der Östrogenund Progesteronrezeptoren kommt dabei keine prädiktive Bedeutung zu [27].

\section{Veränderungen durch Modula- toren des Progesteronrezeptors}

Modulatoren des Progesteronrezeptors wie Mifepriston und Ulipristalacetat können zur Behandlung von Uterusmyomen eingesetzt werden und als Nebeneffekt histopathologische Veränderungen am Endometrium verursachen. Insbesondere kommt es zu zystisch ausgeweiteten
Drüsen mit Sekretionszeichen, sowie Mitosen und „Atypien“ des Drüsenepithels [28]. AEH/EIN-ähnliche Bilder wurden aber nicht beschrieben.

\section{Seröses intraepitheliales Karzinom}

Das seröse intraepitheliale Karzinom (SEIC) wird als Vorläuferläsion des serösen Karzinoms des Endometriums angenommen (• Abb. 11), ist aber gleichzeitig auch Ausdruck einer oberflächlichen Ausbreitung desselben [29]. Ein isoliertes SEIC findet sich selten. Es ist typischerweise mit atrophem Endometrium vergesellschaftet und kann auch in Polypen vorkommen [29]. Das SEIC ist durch hochgradig atypische Zellen mit polymorphen, hyperchromatischen Zellkernen charakterisiert. Durch eine verminderte Kohäsion lösen sich wie bei serösen Tumoren von Tuben und Ovarien Zellkomplexe von der Oberfläche ab. 

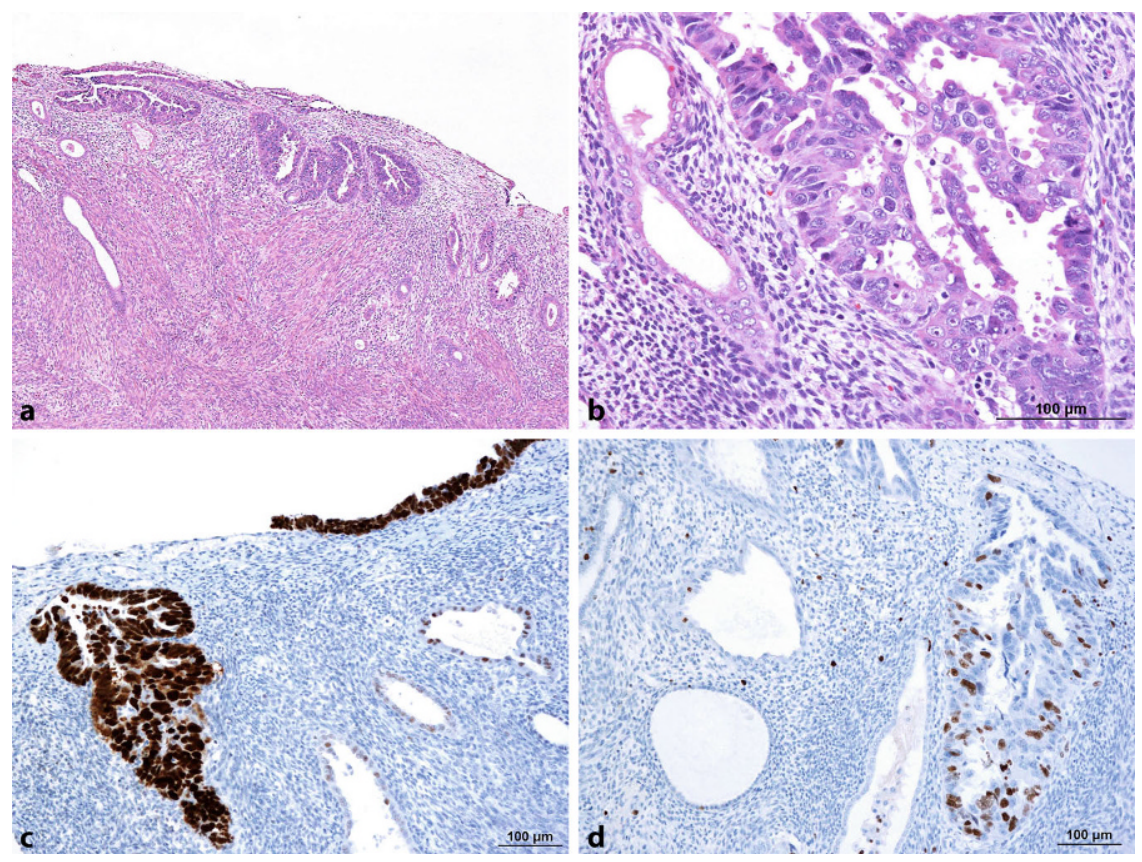

Abb. 11 ॥ Seröses intraepitheliales Karzinom (SEIC) mit flachem Endometrium (a), dessen Oberfläche und Drüsen teilweise von hochgradig atypischem Epithel (b) eingenommen sind. Mutationstypische Immunreaktivität für TP53 (c) und hoher Ki-67-Färbeindex (d). Atypiegrad und unregelmäßige Anordnung der Zellen sprechen gegen AEH/EIN. Auffallend sind einzelne Drüsen mit normalem Epithel. HE-Färbung $(a, b)$, DAB-Färbung $(c, d)$

Mitosen sind reichlich vorhanden und durch atypische Figuren charakterisiert. Immunhistochemisch zeigen sich ein mutationsspezifisches Reaktionsmuster für p53, eine Überexpression von Cyclin $\mathrm{E}$ und ein hoher Ki-67-Färbeindex [30, 31]. Diese Marker sind auch von diagnostischer Relevanz. Auf molekularer Ebene finden sich TP53-Mutationen, allerdings ohne Verlust der Heterozygotie [32]. Das SEIC wird nicht als Vorläuferläsion wie die AEH/EIN eingestuft, sondern als „Oberflächenkarzinom“, da es in einem Teil der Fälle bereits mit einer ausgedehnten Metastasierung im Bereich des Peritoneums sowie mit Läsionen in anderen Teilen des Genitales einhergeht [33]. Für die Entstehung des SEIC und des serösen Karzinoms wird ein von Östrogenen unabhängiger Pfad angenommen. Spezifische Risikofaktoren sind nicht bekannt [34]. Die glanduläre Dysplasie des Endometriums wurde als Vorstufe zum SEIC postuliert, hat sich aber nicht zuletzt aufgrund geringer Reproduzierbarkeit nicht durchgesetzt [2, 35]. Analog zur Tube wurde von einer anderen Gruppe auch im Bereich des Endometriums eine Überexpression von
- Metaplasien sind Ausdruck einer terminalen Differenzierung. Atypische muzinöse Läsionen sind häufig mit Karzinomen assoziiert.

- Für die Therapie einer AEH/EIN mit Gestagenen gibt es kaum Evidenz. Ein verminderter Zellreichtum der Drüsen nach Therapie sagt eine nachhaltige Rückbildung einer AEH/EIN am besten vorher.

- Das seröse intraepitheliale Karzinom (SEIC) ist durch hochgradige Kernpolymorphie, hohen Mitosegehalt und Ablösung von Zellen charakterisiert und kann bereits mit einer extrauterinen Tumoraussaat einhergehen. Diagnostisch hilfreich sind ein mutationspezifisches immunhistochemisches Reaktionsmuster für P53 („Alles oder Nichts") und ein erhöhter Ki67 Färbeindex.

\section{Korrespondenzadresse}

Prim. Univ.-Prof. Dr. S. F. Lax

Institut für Klinische Pathologie und Molekularpathologie, LKH Graz II, Standort West, Akademisches Lehrkrankenhaus der Medizinischen Universität Graz Göstinger Straße 22, 8020 Graz, Österreich sigurd.lax@kages.at dex gefunden [36]. Ein Zusammenhang dieser als p53-Signatur bezeichneten Läsion mit dem SEIC als dessen möglicher Vorläufer wurde nachgewiesen, deren klinische Bedeutung bleibt aber bis dato unklar.

\section{Fazit für die Praxis}

- In der WHO-Klassifikation 2014 der Präneoplasien des Endometriumkarzinoms unterscheidet man nur mehr 2 Kategorien: die Endometriumhyperplasie ohne Atypien (EH) und die atypische Endometriumhyperplasie/endometrioide intraepitheliale Neoplasie (AEH/EIN).

- Für die Diagnosestellung AEH/EIN ist u. a. der Vergleich zum umgebenden Endometrium wesentlich.

- AEH/EIN können eine Reihe genetischer Veränderungen aufweisen wie Inaktivierung von PAX2, PTEN sowie MLH1. Diese können mittels Immunhistochemie nachgewiesen werden und diagnostisch hilfreich sein.
Funding. Open access funding provided by Medical University of Graz.

\section{Einhaltung ethischer Richtlinien}

Interessenkonflikt. S. F. Lax gibt an, dass kein Interessenkonflikt besteht.

Dieser Beitrag beinhaltet keine vom Autor durchge-

Open Access. Dieser Artikel wird unter der Creative Commons Namensnennung 4.0 International Lizenz de) veröffentlicht, welche die Nutzung, Vervielfältigung, Bearbeitung, Verbreitung und Wiedergabe in jeglichem Medium und Format erlaubt, sofern Sie den/die ursprünglichen Autor(en) und die Quelle ordnungsgemäßnennen, einen Linkzur Creative Commons Lizenz beifügen und angeben, ob Änderungen vorgenommen wurden.

\section{Literatur}

1. Lax SF, Kurman RJ (1997) A dualistic model for endometrial carcinogenesis based on immunohistochemical and molecular genetic analyses. Verh Dtsch Ges Pathol 81:228-232 führten Studien an Menschen oder Tieren. (http://creativecommons.org/licenses/by/4.0/deed. 
2. Zaino R, Carinelli SG, Ellenson LH, Eng C, Katabuchi H, Konishi I, Lax S, Matias-Guiu X, Mutter GL, Peters WA III, Sherman ME, Shih I-M, Soslow R, Stewart CJR (2014) Tumors of the uterine corpus. Epithelial tumours and precursors. In: Kurman RJ, Carcangiu ML, Herrington S, Young RH (Hrsg) Tumours of the female reproductive organs. IARC Press, Lyon, $\mathrm{S}$ 125-135

3. Dallenbach-Hellweg G (1987) Histopathology of the endometrium, 4. Aufl. Springer, New York

4. Wright TC, Holinka CF, Ferenczy A, Gatsonis CA, Mutter GL, Nicosia S, Richart RM (2002) Estradiolinduced hyperplasia in endometrial biopsies from women on hormone replacement therapy. Am J Surg Pathol 26(10):1269-1275

5. Lax S (2013) Erkrankungen des Endometriums. In: Dietel M, Klöppel G (Hrsg) Pathologie: Mamma, Weibliches Genitale, Schwangerschaft und Kindererkrankungen, 3. Aufl. Springer, Berlin, Heidelberg, S329-404

6. Kurman RJ, Kaminski PF, Norris HJ (1985) The behavior of endometrial hyperplasia. A long-term study of "untreated" hyperplasia in 170 patients. Cancer 56(2):403-412

7. Mutter GL, Baak JP, Crum CP, Richart RM, Ferenczy A, Faquin WC (2000) Endometrial precancer diagnosis by histopathology, clonal analysis, and computerized morphometry. J Pathol 190(4):462-469

8. Monte NM, Webster KA, Neuberg D, Dressler GR, Mutter GL (2010) Joint loss of PAX2 and PTEN expression in endometrial precancers and cancer. Cancer Res 70(15):6225-6232. https://doi.org/10. 1158/0008-5472.CAN-10-0149

9. Moreno-Bueno G, Hardisson D, Sarrio D, Sanchez C, Cassia R, Prat J, Herman JG, Esteller M, MatiasGuiu X, Palacios J (2003) Abnormalities of E- and $\mathrm{P}$-cadherin and catenin (beta-, gamma-catenin, and p120ctn) expression in endometrial cancer and endometrial atypical hyperplasia. J Pathol 199(4):471-478

10. Baak JP, Mutter GL, Robboy $S$, van Diest PJ, Uyterlinde AM, Orbo A, Palazzo J, Fiane B, Lovslett K, Burger C, Voorhorst F, Verheijen RH (2005) The molecular genetics and morphometrybased endometrial intraepithelial neoplasia classification system predicts disease progression in endometrial hyperplasia more accurately than the 1994 World Health Organization classification system. Cancer 103(11):2304-2312. https://doi. org/10.1002/cncr.21058

11. Trimble $C L$, Kauderer J, Zaino R, Silverberg $S$, Lim PC, Burke JJ 2nd, Alberts D, Curtin J (2006) Concurrent endometrial carcinoma in women with a biopsy diagnosis of atypical endometria hyperplasia: a Gynecologic Oncology Group study. Cancer 106(4):812-819

12. Lacey JV Jr., Mutter GL, Ronnett BM, loffe OB, Duggan MA, Rush BB, Glass AG, Richesson DA, Chatterjee N, Langholz B, Sherman ME (2008) PTEN expression in endometrial biopsies as a marker of progression to endometrial carcinoma. Cancer Res 68(14):6014-6020. https://doi.org/10.1158/00085472.CAN-08-1154

13. Longacre $T A$, Chung $M H$, Jensen $D N$, Hendrickson MR (1995) Proposed criteria for the diagnosis of well-differentiated endometrial carcinoma. A diagnostic test for myoinvasion. Am J Surg Pathol 19(4):371-406

14. Carlson JW, Mutter GL (2008) Endometrial intraepithelial neoplasia is associated with polyps and frequently has metaplastic change. Histopathology 53(3):325-332. https://doi.org/ 10.1111/j.1365-2559.2008.03104.x
15. Moritani S, Kushima R, Ichihara S, Okabe H, Hattori T, Kobayashi TK, Silverberg SG (2005) Eosinophilic cell change of the endometrium: a possible relationship to mucinous differentiation. Mod Pathol 18(9):1243-1248. https://doi.org/10.1038/ modpathol.3800412

16. Lin $M C$, Lomo L, Baak JP, Eng $C$, Ince $T A$, Crum CP, Mutter GL (2009) Squamous morules are functionally inert elements of premalignant endometrial neoplasia.ModPathol 22(2):167-174. https://doi.org/10.1038/modpathol.2008.146

17. Rawish KR, Desouki MM, Fadare O (2017) Atypical mucinous glandular proliferations in endometrial samplings: follow-up and other clinicopathological findings in 41 cases. Hum Pathol 63:53-62. https://doi.org/10.1016/j.humpath.2017.02.002

18. Fadare O, Roma AA, Mhawech-Fauceglia P, Parkash V, Rabban JT (2018) The diagnosis of mucinous lesions in endometrial samplings by gynaecological pathologists: an analysis of diagnostic reproducibility. Pathology 50(3):276-285. https:// doi.org/10.1016/j.pathol.2017.09.014

19. McCluggage WG, McBride HA (2012) Papillary syncytial metaplasia associated with endometrial breakdown exhibits an immunophenotype that overlaps with uterine serous carcinoma. Int J Gynecol Pathol 31(3):206-210. https://doi.org/10 1097/PGP.0b013e31823bb1a1

20. Simon RA, Peng SL, Liu F, Quddus MR, Zhang C, Steinhoff MM, Lawrence WD, Sung CJ (2011) Tuba metaplasia of the endometrium with cytologic atypia: analysis of p53, Ki-67, TERT, and long-term follow-up. Mod Pathol 24(9):1254-1261. https:// doi.org/10.1038/modpathol.2011.78

21. Nucci MR, Prasad CJ, Crum CP, Mutter GL (1999) Mucinous endometrial epithelial proliferations: a morphologic spectrum of changes with diverse clinical significance. Mod Pathol 12(12):1137-1142

22. Park CK, Yoon G, Cho YA, Kim HS (2016) Clinicopathological and immunohistochemical characterization of papillary proliferation of the endometrium: A single institutional experience. Oncotarget 7(26):39197-39206. https://doi.org/ 10.18632/oncotarget.10049

23. Lucas $\mathrm{E}$, Chen $\mathrm{H}$, Molberg $\mathrm{K}$, Castrillon $\mathrm{DH}$, Rivera Colon G, Li L, Hinson S, Thibodeaux J, Lea J, Miller DS, Zheng W (2018) Mismatch repair protein expression in endometrioid Intraepithelial neoplasia/atypical Hyperplasia: should we screen for lynch syndrome in precancerous lesions? Int J Gynecol Pathol. https://doi.org/10.1097/PGP. 0000000000000557

24. Kurman RJ, Norris HJ (1982) Evaluation of criteria for distinguishing atypical endometrial hyperplasia from well-differentiated carcinoma. Cancer 49(12):2547-2559

25. Emons G (2018) S3-Leitlinie Diagnostik, Therapie und Nachsorge der Patientinnen mit Endometriumkarzinom (Langversion). Therapie der Präkanzerosen und des frühen Endometriumkarzinoms, $S$ 94-107

26. Wheeler DT, Bristow RE, Kurman RJ (2007) Histologic alterations in endometrial hyperplasia and well-differentiated carcinoma treated with progestins. Am J Surg Pathol 31(7):988-998. https://doi.org/10.1097/PAS.0b013e31802d68ce

27. Gunderson CC, Dutta S, Fader AN, Maniar KP, Nasseri-Nik N, Bristow RE, Diaz-Montes TP, Palermo R, Kurman RJ (2014) Pathologic features associated with resolution of complex atypical hyperplasia and grade 1 endometrial adenocarcinoma after progestin therapy. Gynecol Oncol 132(1):33-37. https://doi.org/10.1016/j.ygyno.2013.11.033
28. Mutter GL, Bergeron C, Deligdisch L, Ferenczy A, Glant M, Merino M, Williams AR, Blithe DL (2008) The spectrum of endometrial pathology induced by progesterone receptor modulators. Mod Pathol 21(5):591-598. https://doi.org/10. 1038/modpathol.2008.19

29. Ambros RA, Sherman ME, Zahn CM, Bitterman P, Kurman RJ (1995) Endometrial intraepithelial carcinoma: a distinctive lesion specifically associated with tumors displaying serous differentiation. Hum Pathol 26(11):1260-1267

30. Sherman ME, Bur ME, Kurman RJ (1995) p53 in endometrial cancer and its putative precursors: evidence for diverse pathways of tumorigenesis. Hum Pathol 26(11):1268-1274

31. Kuhn E, Bahadirli-Talbott A, Shih le M (2014) Frequent CCNE1 amplification in endometrial intraepithelial carcinoma and uterine serous carcinoma. Mod Pathol 27(7):1014-1019. https:// doi.org/10.1038/modpathol.2013.209

32. Tashiro $\mathrm{H}$, Isacson C, Levine R, Kurman RJ, Cho KR, Hedrick L (1997) p53 gene mutations are common in uterine serous carcinoma and occur early in thei pathogenesis. Am J Pathol 150(1):177-185

33. WheelerDT, Bell KA, Kurman RJ, Sherman ME (2000) Minimal uterine serous carcinoma: diagnosis and clinicopathologic correlation. Am J Surg Pathol 24(6):797-806

34. Sherman ME (2000) Theories of endometrial carcinogenesis: a multidisciplinary approach. Mod Pathol 13(3):295-308. https://doi.org/10.1038/ modpathol.3880051

35. Zheng W, Liang SX, Yi X, Ulukus EC, Davis JR, Chambers SK (2007) Occurrence of endometrial glandular dysplasia precedes uterine papillary serous carcinoma. Int J Gynecol Pathol 26(1):38-52. https://doi.org/10.1097/01.pgp. $0000228138.56222 .4 \mathrm{e}$

36. Jarboe EA, Pizer ES, Miron A, Monte N, Mutter GL, Crum CP (2009) Evidence for a latent precursor ( $\mathrm{p} 53$ signature) that may precede serous endometrial intraepithelial carcinoma. Mod Pathol 22(3):345-350. https://doi.org/10. 1038/modpathol.2008.197 